\title{
Using prime numbers for automatic theorem proving
}

\author{
Open Mathematics Collaboration*广
}

August 7, 2020

\begin{abstract}
We apply an analogous setting from Gödel's numbering system to automatic theorem proving.
\end{abstract}

Gödel's numbering, automatic theorem proving, artificial intelligence

The most updated version of this paper is available at https://osf.io/g7usc/download

\section{Introduction}

1. The strategies in this work mimic Gödel's numbering system [1].

2. $P=$ set of prime numbers

3. $C=$ set of composite numbers

4. $P=\{2,3,5,7,11,13,17,19,23,29,31, \ldots\}$

5. $C=\{4,6,8,9,10,12,14,15, \ldots\}$

*All authors with their affiliations appear at the end of this paper.

†Corresponding author: mplobo@uft.edu.br | Join the Open Mathematics Collaboration 


\section{Notation \& Definition}

6. If $S$ is a set, then $S[i]$ is the $i$-th element of $S$.

7. Example: $P[3]=5$.

8. $x \in X$ means $p \in P, c \in C, \ldots$

9. In some family of sets, $\mathcal{Y}[i]=0$ means "no" and $\mathcal{Y}[j]=1$ means "yes".

10. $(\mathcal{Y}[i]=0) \equiv(\nexists y \in \mathcal{Y}[i])$

11. $(\mathcal{Y}[j]=1) \equiv(\exists y \in \mathcal{Y}[j])$

\section{Axioms}

12. $A=P=$ set of axioms

13. $A[i]=i$-th axiom

\section{Theorems}

14. $T=C=$ set of definitions and theorems

15. In the following, some examples are provided for the meaning of $T\left[p_{i}\right]$.

16. $T\left[p_{j}\right] \equiv$ definition of simple groups

17. $T\left[p_{k}\right] \equiv$ definition of normal subgroups

18. $T\left[p_{l}\right] \equiv$ definition of abelian elements

19. $T\left[p_{m}\right] \equiv$ Any non-abelian group of order 6 is isomorphic to $S_{3}$ 


\section{Exponents}

20. $E=P=$ set of strategies performed in theorem proving

21. In the following, some examples are provided for the meaning of the prime exponents.

22. $E[1]=2=$ there exist both abelian and non-abelian cases

23. $E[2]=3=$ check if there are subcases in each case

24. $E[3]=5=$ use contrapositive law

\section{Computer (artificial intelligence)}

25. Let $g=(3 \cdot 5)^{7 \cdot 11 \cdot 37}$.

26. $g$ means the strategies 7,11 , and 37 were applied in the theorem 15 , which is a combination of the axioms 3 and 5 .

\section{Final Remarks}

27. Automatic theorem proving can be transformed into arithmetic calculations in a machine.

\section{Open Invitation}

Review, add content, and co-author this paper [2,3]. Join the Open Mathematics Collaboration (https://bit.ly/ojmp-slack). Send your contribution to mplobo@uft.edu.br. 


\section{Open Science}

The latex file for this paper together with other supplementary files are available [4].

\section{Ethical conduct of research}

This original work was pre-registered under the OSF Preprints [5], please cite it accordingly [6]. This will ensure that researches are conducted with integrity and intellectual honesty at all times and by all means.

\section{Acknowledgement}

+ Center for Open Science https://cos.io

+ Open Science Framework https://osf.io

\section{References}

[1] Nagel, Ernest, James R. Newman, and Douglas R. Hofstadter. Gödel's Proof. NYU Press, 2001.

[2] Lobo, Matheus P. "Microarticles." OSF Preprints, 28 Oct. 2019. https://doi.org/10.31219/osf.io/ejrct

[3] Lobo, Matheus P. "Simple Guidelines for Authors: Open Journal of Mathematics and Physics." OSF Preprints, 15 Nov. 2019. https://doi.org/10.31219/osf.io/fk836

[4] Lobo, Matheus P. "Open Journal of Mathematics and Physics (OJMP)." OSF, 21 Apr. 2020. https://doi.org/10.17605/osf.io/6hzyp 
[5] COS. Open Science Framework. https://osf.io

[6] Lobo, Matheus P. "Using Prime Numbers for Automatic Theorem Proving." OSF Preprints, 4 July 2020. https://doi.org/10.31219/osf.io/g7usc

\section{The Open Mathematics Collaboration}

Matheus Pereira Lobo (lead author, mplobo@uft.edu.br) $)^{1,2}$ https://orcid.org/0000-0003-4554-1372

${ }^{1}$ Federal University of Tocantins (Brazil)

${ }^{2}$ Universidade Aberta (UAb, Portugal) 